DOI 10.18699/PlantGen2019-023

\title{
Expression of sheep pox viral A27L and L1R proteins in prokaryotic and eukaryotic systems
}

\author{
Beisenov D.K. ${ }^{1 *}$, Stanbekova G.E. ${ }^{1}$, Karimov N.Zh. ${ }^{2}$, Iskakov B.K. ${ }^{1}$ \\ ${ }^{1}$ M. Aitkhozhin Institute of Molecular Biology and Biochemistry, Almaty, Kazakhstan \\ ${ }^{2}$ Kazakh Scientific Research Institute of Animal Breeding and Forage Production, Almaty, Kazakhstan \\ *e-mail: daniyar.b@mail.ru
}

Sheep pox is a severe illness causing mass death among small ruminants. Epizootics in different countries including Republic of Kazakhstan leads to great economic loses. Sheep pox virus (SPPV) belongs to Capripoxvirus genus of the Poxviridae family. Attenuated strains are used as vaccines for prophylactic means of the disease. Such vaccines are effective but has crucial disadvantage as virions could revert to virulent form by recombination. Genome sequencing and genetic engineering methods give opportunities to produce save vaccine with less expense. Nucleotide sequence of the SPPV "NISKHI" strain used in our work was analyzed to find orthologs of the immunogenic structural proteins of vaccinia virus. sppv-niskhi-117 gene coding A27L ortholog (17.3 kDA) and sppv-niskhi-060 gene coding L1R ortholog (20.3 kDA) were used for expression in various systems. Genes were cloned in bacterial pET-19b expression vector. Bacterially produced (E. coli BL21 strain) and purified proteins were used for rabbit immunization. Antibodies raised to recombinant proteins showed virus neutralizing activity. pACT2 vector was used to express proteins in Saccharomyces cerevisiae cells (CG 1945 strain). Only A27L protein successfully expressed in yeast cells. Transgenic Nicotiana tabacum and Brassica napus plants expressing viral proteins were obtained after transformation by pCambia 2300 vectors containing viral genes, translational enhancers and sequences for signal peptides to target recombinant proteins into chloroplast or endoplasmic reticulum. A higher recombinant protein yield was achieved when using transient expression (magnification) in Nicotiana benthamiana leaves. Maximal viral protein level was obtained in transplastomic N. tabacum plants carrying viral genes in the chloroplast genome. L1R protein tend to form di- and trimeric forms in E. coli and plants. Synthesized proteins may be used for development efficient recombinant subunit vaccine against sheep pox. 